\title{
An efficient design approach of ROI based DWT using vedic and wallace tree multiplier on FPGA platform
}

\author{
Vijaya S.M' ${ }^{1}$, Suresh $K^{2}$ \\ ${ }^{1}$ Rajarajeswari College of Engineering (RRCE), India \\ ${ }^{2}$ SEA College of Engineering \& Technology, India
}

\begin{abstract}
Article Info
Article history:

Received Aug11, 2018

Revised Mar 1, 2019

Accepted Mar 12, 2019

\section{Keywords:}

Adder-tree

DWT

FPGA

Matlab

MRI

MSE

PSNR

ROI

Vedic Multiplier

Wallace-tree Multiplier

ABSTRACT

In digital image processing, the compression mechanism is utilized to enhance the visual perception and storage cost. By using hardware architectures, reconstruction of medical images especially Region of interest (ROI) part using Lossy image compression is a challenging task. In this paper, the ROI Based Discrete wavelet transformation (DWT) using separate Wallace- tree multiplier (WM) and modified Vedic Multiplier (VM) methods are designed. The Lifting based DWT method is used for the ROI compression and reconstruction. The 9/7 filter coefficients are multiplied in DWT using Wallace- tree multiplier (WM) and modified Vedic Multiplier (VM). The designed Wallace tree multiplier works with the parallel mechanism using pipeline architecture results with optimized hardware resources, and 8x8 Vedic multiplier designs improves the ROI reconstruction image quality and fast computation. To evaluate the performance metrics between ROI Based DWT-WM and DWT-VM on FPGA platform. The PSNR and MSE are calculated for different Brain MRI images, and also hardware constraints include Area, Delay, maximum operating frequency and power results are tabulated. The proposed model is designed using Xilinx platform using Verilog-HDL and simulated using ModelSim and Implemented on Artix-7 FPGA device.
\end{abstract}

Copyright $@ 2019$ Institute of Advanced Engineering and Science. All rights reserved.

\section{Corresponding Author:}

Vijaya S.M,

Rajarajeswari College of Engineering (RRCE),

Bengaluru, India.

Email: vijaya@rrce.org

\section{INTRODUCTION}

Medical imaging place critical role in today's health care of patients for entire treatment process from diagnostics to surgical operations. Medical image processing has to face a lot of challenges in image management, data mining, Medical visualization for Virtual Reality (VR) bio-imaging and Neuro-imaging. The amount of medical image data is increasing from kilo to terabyte storage due to Image resolution and visualization, and it is costly [1]. Image compression techniques are established to reduce the number of bits which is required to transmit or store images without loss of data information. Compressed image minimizes the expense bandwidth and cost-effective during image transmission. In today's world, the application fields for such compressions include Mobiles, TV, Video broadcasting with High Definition (HD), digital video recording and so on [2]. The fundamental components of image compression are redundancy reduction which removes the image duplication while irrelevancy reduction removes unwanted partsfrom the image, which is not observed in receiver section [3].

The extensively used image compression techniques can be categorizing into two parts lossless and Lossy compression. Lossless compression which recovers the input image after decompression from the compressed image. In Lossy compression, An image must be reconstructed after decompression with the acceptable loss of quality. Many compression algorithms are developed by researchers to compress and 
rebuild the image with high quality. The image compression techniques like Discrete Wavelet Transformation (DWT), Fast Fourier transformation (FFT), DCT (Discrete cosine transformation), Sparse based Wavelet, Singular Value Decomposition (SVD) and Block Truncation Coding (BTC) are used for enhancement of image quality and compression ratio [4-6]. The effective lossless compression techniques include Huffman Encoding (HE), Run Length Encoding (RLE), Arthematic Encoding and Dictionary Techniques (LZ77, LZ78, LZW) and bit plane coding [7].To improve the compression ratio, Quality of the image, hybrid algorithms are designed which are the combination of two or more compression techniques. Hybrid compression technique [8] introduces to enhance the standard of the reconstructed image with high PSNR value which includes the combination of Principal Component Analysis (Also known as KarhunenLoeveTransform (KLW)), DCT followed by SPIHT technique (Set-Partitioning-In-Hierarchical-Trees) and results to minimize the encoding time.

ROI Based hybrid compression algorithms to improve the PSNR, CR, Space saving and timeconsuming compared to conventional approaches. The ROI based lossless image compression with Embedded Zero-tree Wavelet (EZW) Algorithm [9] is designed for better diagnosis and compared with other techniques like Haar Wavelet (HW) [10] and Daubechies wavelet (DbW) Transformation with better image quality. The Burrows-Wheeler (BW) Transform and Move to Front(MTF) transform with Huffman encoding and hybrid fractal encoding [11] and DWT followed by SPHIT technique [12] are ROI based hybrid compression approach applicable to medical MRI images with CR improvements. The ROI of radiological Image applied to Vedic compression algorithm [13] on software approach with improvement in computation speed, compression ratio and visual quality outcomes with different image database over conventional DCT approach. The FPGA based approach with 2D-DWT using modified Vedic Multiplier [14] with less number of Half and full adder in adder- tree module which results in less resource but the output image quality is not up to the standards.

Most of the Research work towards on ROI-Based image compression with Lossy or lossless techniques are software-based approaches. Very few works on hardware-based procedures for Medical image processing with optimization problems, Hardware complexity, and Performance degradation with constraints. Our proposed design towards ROI Based Lifting scheme DWT approach using Vedic Multiplier to improve the computation speed with image quality image and Wallace tree multiplier enhances the hardware complexity with optimization using pipelining architectures on FPGA. Section 1 has discussed the existing approaches using ROI image compression, FPGA based DWT methods with Vedic multiplier and Wallace tree multipliers. In Section 3, Overview of the methodology which deals with the proposed design. The ROI Based DWT-WM and VM design methods explained in detail in section 4 followed by the results and discussion with performance tabulation in section 5, and section 6 gives the conclusion of the overall work.

This section discusses the methods adopted for ROI based image compression for different medical images, DWT Approaches, Vedic and Wallace multiplier during recent years. Hamzah et al. [15] have presented the Four-dimensional- ROI Image compression and used non-separable Double Lifting integer wavelet transform (IWT) to reduce the rounding noise, and the number of rounding operators along with increasing coding performance and the architecture is compatible to JPEG-2000 conventional approach. The Blocked ROI compressive sensing (CS) of THEMIS images and its reconstruction of images with improvement in image quality and CR has presented by Srija et al. [16]. Amit et al. [17] have given a Polygonal ROI based image compression of DICOM images for telemedicine applications using zero padding, Lossy, and lossless wavelet compression. For Better Portable Graphics (BPG), David et al. [18] have presented an ROI based image compression techniques which include video coding algorithms and results $25 \%$ improvement in CR over traditional image compression techniques. The cloud-based solutions for histopathological images using ROI based compression are presented by Aparna et al. [19] with great CR with optimized storage to and from the cloud.

To perform the ROI compressions from the current survey, suitable hardware based transformations are selected to show the image compression. Kiranmaye et al. [20] have presented a 2D-DWT lifting approach having overlapped-stripe based scanning method which reduces the temporary memory in the process and predicts, updates and addition models are incorporated in architecture and results with less area and power consumption than previous approaches, but lags with image quality and computation speed. For error tolerant applications, the 2D-DWT method followed by a smooth Gaussian filter is designed by Nayna et al. [21] which results in the average image quality output which is acceptable to human eyes. Shweta et al. [22] have presented DWT for image compression using Mallat algorithm structure on Xilinx system generator and which results from right reconstruct image and lags with an area and power consumption on FPGA.

To improve the computation speed, multipliers place critical role to maintain the compression and quality of reconstructed images. Gadakh et al. [23] have presented Ancient 16 X 16 Vedic Multiplier (VM)includes Urdhva-Tiryagbhyam (UT) sutra and results with path delay and memory consumption, 
but it is same as conventional approaches. The $8 \times 8$ Vedic multiplier with modified Full adders presented by Bandi et al. [24] with performance analysis of Area resource. Kahar et al. [25] show a 64 X64 Vedic multiplier with conventional approaches and performance include area and path delay are tabulated. This multiplier uses the same traditional approaches without novelty. The Xuan et al. [26] has presented an unsigned 32-bit multiplier using Wallace multiplier and booth encoder. The Carry-lookahead adder and modified Wallace tree adder used as adder-tree and which gives fast results with more area utilization. Asif et al. [27] present a Wallace-tree (WM) multiplier includes high-speed counters. Lucas et al. [28] and Ram et al. [29] presents Wallace multipliers using Carry select adder with an area and delay comparison.

Sunaina et al. [30] and Suma et al. [31] presents the Vedic multiplier based image compression using DWT method which includes conventional Vedic approach and traditional DWT approaches and results from the area and delays utilization tabulation with no improvement in image quality. The work of Na'am et al. [32] have concentrated on medical image processing of fundus image and introduced an automatic ROI mechanims that adopts threshold method of segmentation. Further, an work of Lakshminarayana and Sarvagya [33], presented an compresive sensing based medical image compression by considering ROI with lossless compression. The perfromance of [33] is analyzed by considering MSE, PSNR and compression ratio.

There is a lot of study towards Image compression using Transformation and other methods as software approaches and fewer hardware approaches in medical image applications. Very few works on ROI based criteria for Medical images using the Vedic multiplier based on FPGA with lagging in performance matrices includes PSNR, MSE and resource optimization. These problems are overcome in proposed techniques with the following section.

\section{PROPOSED METHODOLOGY}

The proposed design includes ROI Based Lifting scheme of discrete wavelet Transformation (DWT) using area efficient Wallace- tree multiplier (WM) and modified Vedic Multiplier (VM). The Wallace tree multiplier works with parallel processing using pipelined architecture results in an optimized area with high speed. The modified Vedic Multiplier is used to enhance the execution speed of the proposed design. The Figure 1 represents the architectural model of the proposed plan.

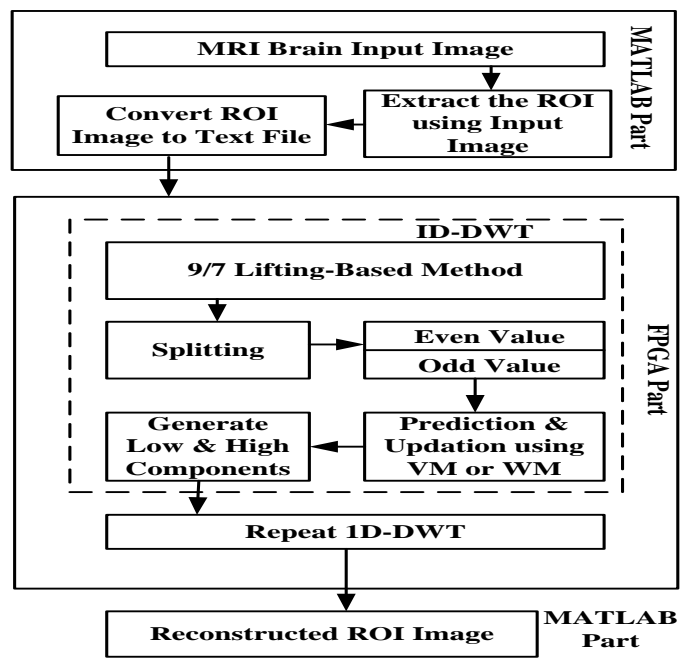

Figure 1. Proposed methodology

Consider the MRI-Brain Input image, extract the ROI part from the input image, and convert the ROI Image part to text file format using Matlab. The proposed design is applicable only to ROI image part and not to complete MRI-brain image. The 9/7 lifting scheme based DWT receives the text file through testcases. Split the ROI image pixels to even and odd components. The prediction followed by updating is performing along with Multiplication of 9/7 lifting coefficients using Wallace-tree or Vedic Multiplier. The low and high pass components are generated and repeat the same process to reconstruct the ROI image. In the next section, a detailed discussion of the Design and working is elaborated. 


\section{PROPOSED DESIGN}

The proposed design is to perform the ROI image compression using Wallace-tree and Vedic Multiplier. The following Figure 2, indicates the hardware representation of proposedlifting scheme based 1D-DWT using WM or VM. Compare to complete MRI-Brian image, ROI portion image compression has an enormous advantage with fast computation, less resource utilization, and less hardware complexity over FPGA scenario.

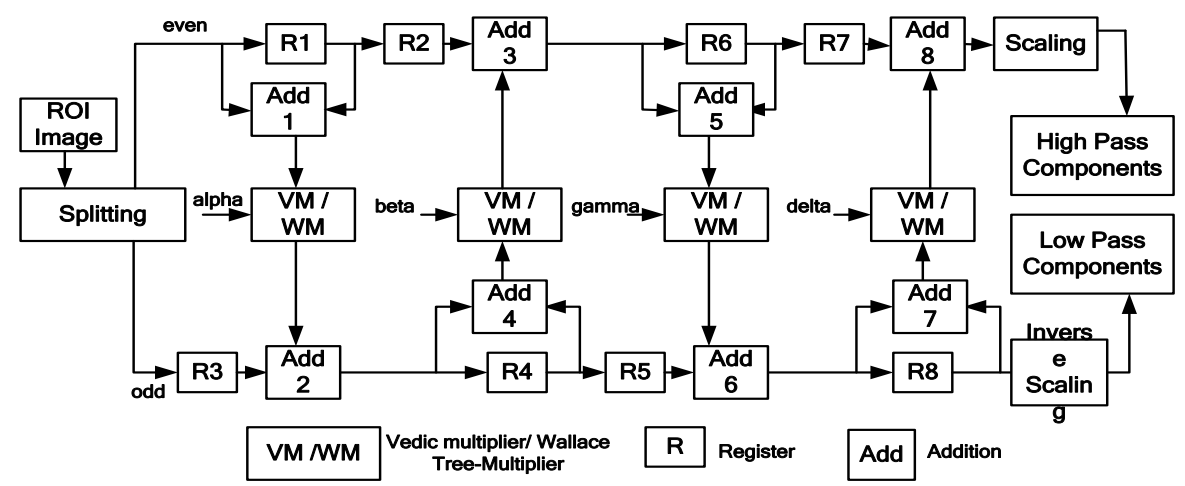

Figure 2. The hardware representation of proposed lifting scheme based 1D-DWT using WM or VM

The ROI image pixels are stored in memory locations based on sizes as a text format. Extract the pixels serially and split to even and odd components-based clock. The design uses 8-Registers, 8- addition units and 4- multiplication unit using WM or VM which are part of the prediction and update operation followed by scaling and inverse scaling. The prediction operation includes the even components are added (Add1) with delayed register (R1), the outcomes are multiplied (WM/VM) with one of the Daubechies 9/7 "alpha" filter coefficients. The updating operation includes the odd components after added with predicated output, perform the addition with odd delayed components, then multiply the "beta" coefficient the process repeats for "gamma" and "delta" coefficients as a prediction and updates operations respectively. Similarly, Scaling and inverse scaling modules are performed using prediction and update activities with scaling and inverse scaling factors to generate the high and low pass components respectively. The 1D-DWT outputs include low and high pass components are fed to the DWT process again, to create the 2D- DWT output components, which are used to recover back and reconstruct the ROI Image. The Filter coefficient multiplication is done using WM and VM separately in DWT module are explained in detail with following architectures. The proposed Design ROI Based DWT Architecture uses two different Multiplier methods namely Wallace tree multiplier and Vedic Multiplier. The practical operation of both the multipliers is explained below.

\subsection{Wallace tree multiplier}

The pipeline architecture of Wallace- tree Multiplier is designed and shown in Figure 3. It mainly includes Booth encoder using Radix-4 Booth recording table, Partial product generation, Compressors, and adder- tree Module. The booth encoder uses the 8-bit multiplier input for booth compression using radix4booth recording is tabulated in Table-1. Based on Multiplier input, the encoded record includes 0,1, -1, 2 and -2 is multiplied with 8 -bit multiplicand for partial product formation. The partial product generates the output and complements based on multiplicand.

Table 1. Radix-4 booth recording

\begin{tabular}{ccc}
\hline B(Multiplier) & Recording & Partial Product (PP) \\
\hline 000 & 0 & 0 \\
001 & 1 & 1XMultiplicand \\
010 & 1 & 1XMultiplicand \\
011 & 2 & 2XMultiplicand \\
100 & -2 & -2 XMultiplicand \\
101 & -1 & $-1 X$ Xultiplicand \\
110 & -1 & -1 XMultiplicand \\
111 & 0 & 0 \\
\hline
\end{tabular}




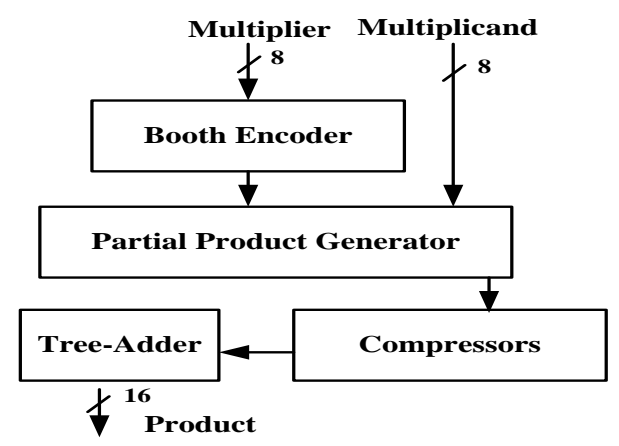

Figure 3. Wallace-tree Multiplier Design

The partial product outputs used in four compressors to reduce the number of stages of multiplication operations to boost the computation process and inputs to the Wallace tree adder. The Wallace tree adder includes the half adder (HA), the full adder (FA), FA with carrying generation along with oneinverted and two- inverted inputs. The prevention of sign extension also included in the design of Wallacetree adder option. The tree-adder results from the 16-bit product as a final output.

\subsection{Vedic multiplier}

The proposed 8x8 modified Vedic multiplier operates multiply two decimal or binary number in less time according to the Urdhva Tiryakbhyam sutra. This sutra manages the inputs vertically and crosswise. Firstly, design $2 \times 2$ Vedic multiplier using two half-adder concerning vertical and crosswise operations. To design the $4 \times 4$ Vedic multiplier, four $2 \times 2$ Vedic multipliers along with 4-bit adder and two 6-bit adders used. Similarly, to develop 8X8 Vedic Multiplier, four 4X4 Vedic Multiplier, one 8-bit adder and two 12-bit adders are used which is as shown in Figure 4.

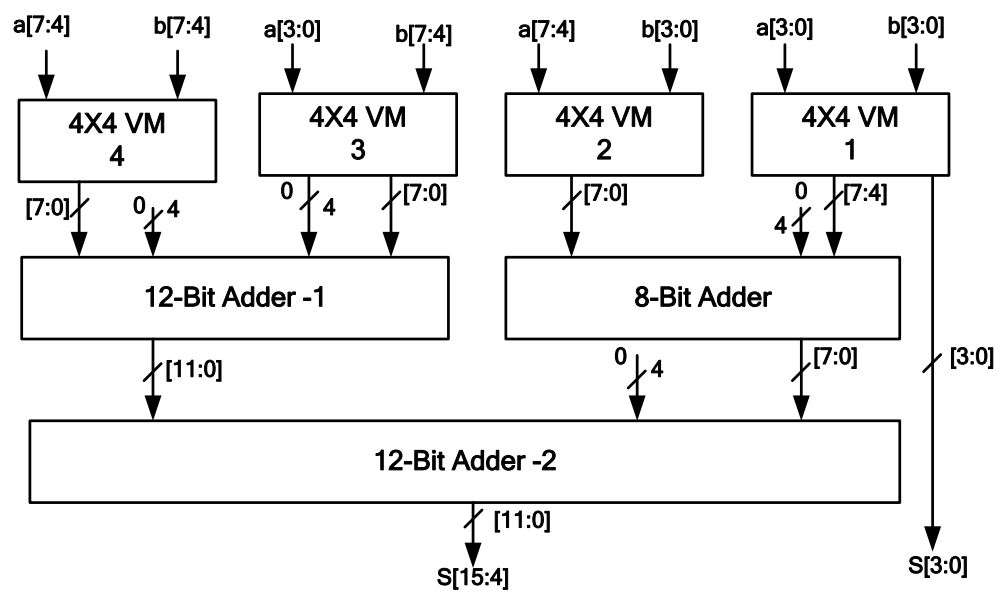

Figure 4. Proposed 8X8 Vedic multiplier

Apply the two 8-bit inputs (a7: a0, b7: b0) to four 4x4 Vedic multipliers vertical and cross-wisely. The first 4x4 VM results from LSB 4-bit as a final sum of first 4-bits (s [3:0]). For 8-bit adder receives second 4x4 VM as first 8-bit input and first 4x4 VM's MSB 4-bits [7:4] along with four zeros' as a second 8-bit input. Similarly, for the 12-bit adder, third 4x4 VM 8-bit output along with four zeros (LSB side) as first 12-bit input and forth 4x4 VM 8-bit output along with four zeros (MSB side) as second 12-bit input results 12-bit adder output. Further, final 12-bit adder requires 8-bit adder output along with four zero's (MSB side) as 12-bit input and first 12-bit adder output as the second input after addition, Results from the 12-bit output which considered as final output (s [15:4]). 


\section{RESULTS AND ANALYSIS}

The proposed Lifting based DWT using Wallace-tree Multiplier (DWT-WM) and Vedic Multiplier (DWT-VM) results analyzed in the below section. The entire model is designed over Xilinx ISE 14.7 Platform using Verilog-HDL and simulated using Modelsim 6.5 simulator and prototyped on FPGA includes Artix-7 family, XC7A100T-3 device, with package CSG324.

The obtained results from the proposed modelover the FPGA platform represented in igure 5. By using Matlab, convert the 256x 256 Brain MRI-Input image-5(a) into text file format. Apply Text file through test case of the proposed design and simulate using a ModelSim simulator, store the low and high pass components in output text file, convert back image format using Matlab to generate the 1D-DWT output image. Compare to DWT-WM, DWT-VM gives better image resolution and quality shown in Figure 5(b) and $5(\mathrm{c})$ respectively.

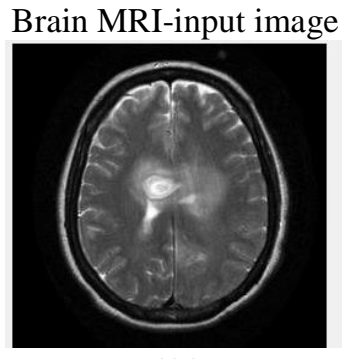

$5(a)$

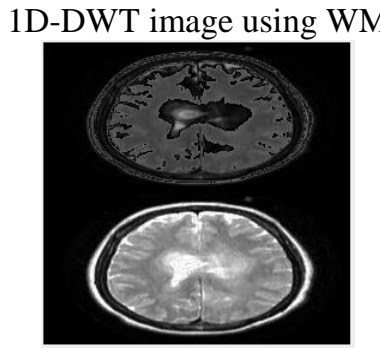

5 (b)

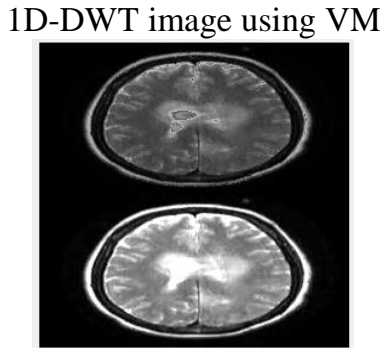

$5(\mathrm{c})$

Figure 5. 5(a) Brain MRI-input image, 5(b) 1D- DWT using Wallace-tree Multiplier (WM), 5(c) 1D- DWT using Vedic Multiplier (VM) Results on FPGA Platform

The ROI- Based DWT Design using WM and VM simulation results are shown in Figure 6 and Figure 7 respectively. For both the simulation, 90 x 86 size of ROI Image is considered. Once clock (Clk) activated with active low asynchronous reset (rst) signal, the process starts, and the ROI image includes 8-bit pixel information stored in allocated 7740 memory locations. The memory location (mem_adrs) divides into 3870 address for even and odd inputs after splitting. Perform DWT operation, the 8-bit low (lout) and high (hout) pass components generated.

\begin{tabular}{|c|c|c|c|c|c|c|c|c|c|c|c|c|c|c|c|c|}
\hline clk & 0 & & 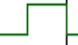 & 1 & t & 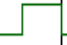 & 1 & e & ] & 1 & 」 & 工 & & & & \\
\hline rst & 0 & & & & & & & & & & & & & & & \\
\hline even_in & $3 f$ & $\begin{array}{ll}66 & 458 \\
6\end{array}$ & $\sqrt{34}$ & & $\sqrt{2 c}$ & $\sqrt{2 d}$ & 20 & $3 c$ & 40 & $\sqrt{2 f}$ & $\sqrt{67}$ & 62 & 30 & $\sqrt{3 e}$ & $\sqrt{40}$ & $\sqrt{3 f}$ \\
\hline odd_in & 46 & $79 \quad 30$ & $3 a$ & $3 b$ & 32 & 22 & 29 & $\sqrt{4 e}$ & 34 & $\sqrt{43}$ & 77 & 42 & 35 & $\sqrt{43}$ & $3 c$ & 46 \\
\hline lout & 029 & 043040 & 2043 & & $\sqrt{040}$ & Jo3c & 工027 & (045 & Co39 & 020 & 022 & Jo1e & Co1b & 012 & 027 & 7029 \\
\hline hout & 005 & obe & & & & Jo08 & $\sqrt{003}$ & 3018 & 000 & $\sqrt{008}$ & $\sqrt{00 a}$ & Co07 & 工我1 & 5003 & Jooe & 2005 \\
\hline mem_adrs & 3870 & 3855 & 3857 & 3858 & 3855 & 3866 & 3861 & 3866 & $386 \beta$ & 3864 & 3865 & 386 & 3867 & $386 \beta$ & 3869 & 3876 \\
\hline
\end{tabular}

Figure 6. Simulation Results of ROI-Based DWT Design using Wallace-Tree Multiplier

\begin{tabular}{|c|c|c|c|c|c|c|c|c|c|c|c|c|c|c|c|c|}
\hline lclk & 0 & & & & & & & & & & 」 & & & & & \\
\hline irst & 0 & & & & & & & & & & & & & & & \\
\hline leven_in & $3 f$ & \begin{tabular}{|ll}
$6 \mathrm{~b}$ & 58 \\
\end{tabular} & $\sqrt{34}$ & & $\sqrt{2 c}$ & $\sqrt{2 d}$ & $\sqrt{20}$ & $\sqrt{3 c}$ & 40 & $2 f$ & $\sqrt{67}$ & $\sqrt{62}$ & 30 & $\sqrt{3 e}$ & 40 & $3 f$ \\
\hline dd_in & 46 & \begin{tabular}{|l|l|l}
79 & 30 \\
\end{tabular} & $\sqrt{3 a}$ & $\sqrt{3 b}$ & $\sqrt{32}$ & $\sqrt{22}$ & $\sqrt{29}$ & $\sqrt{4 e}$ & 34 & 43 & 77 & 42 & 35 & 43 & $\sqrt{3 c}$ & $\sqrt{46}$ \\
\hline lout & $06 \mathrm{c}$ & $0 \mathrm{bb}$ & Job6 & & lob2 & logd & $\sqrt{083}$ & loas & 096 & $\sqrt{065}$ & $\sqrt{05 e}$ & 057 & (04a & $\sqrt{043}$ & $\sqrt{063}$ & 1060 \\
\hline hout & 03d & 060 & 工o63 & & & $05 c$ & Co4d & $\sqrt{04 \mathrm{e}}$ & 060 & $\sqrt{03 f}$ & $\sqrt{036}$ & 032 & $02 \mathrm{~d}$ & $\sqrt{024}$ & $\sqrt{02 b}$ & $03 \mathrm{~d}$ \\
\hline em_adrs & 3870 & 855 & 3857 & $\sqrt{38}$ & $\sqrt{3855}$ & $\sqrt{3860}$ & 3866 & 3860 & 386 & 386 & $\sqrt{3865}$ & 3866 & 386 & 3866 & 3869 & 387 \\
\hline
\end{tabular}

Figure 7. Simulation Results of ROI-Based DWT Design using Vedic Multiplier 
The ROI- Based DWT-WM and DWT-VM Results on FPGA are represented in Figure 8. The different 256x256 input MRI brain images (8a) is considered, select the ROI part of image1 with size 86x90, image 2 with size $90 \times 90$ and image 3 with size 90x86 from the input images (8b) perform the DWT operation using WM and VM, recover the output ROI images, which is same as input ROI image shown in 8(c) and 8 (d) respectively. To investigate the performance metrics of the recovered ROI image quality, PSNR and MSE taken into considerations.

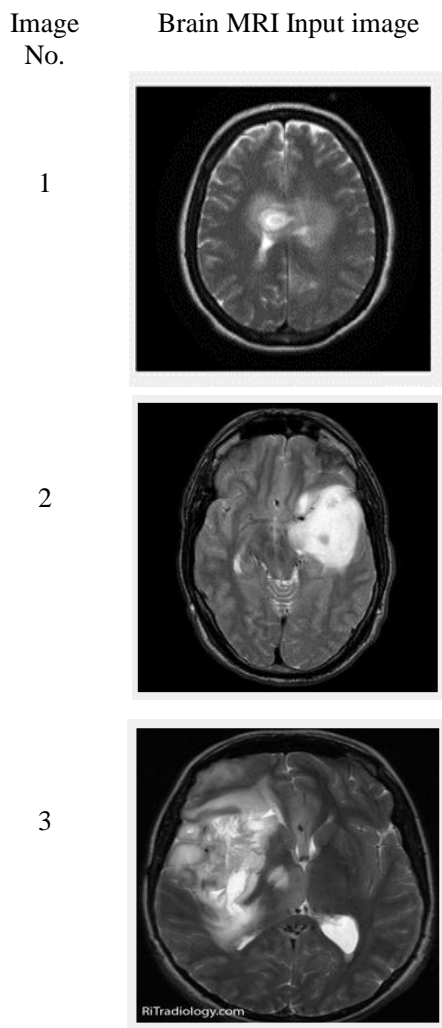

$8(\mathrm{a})$
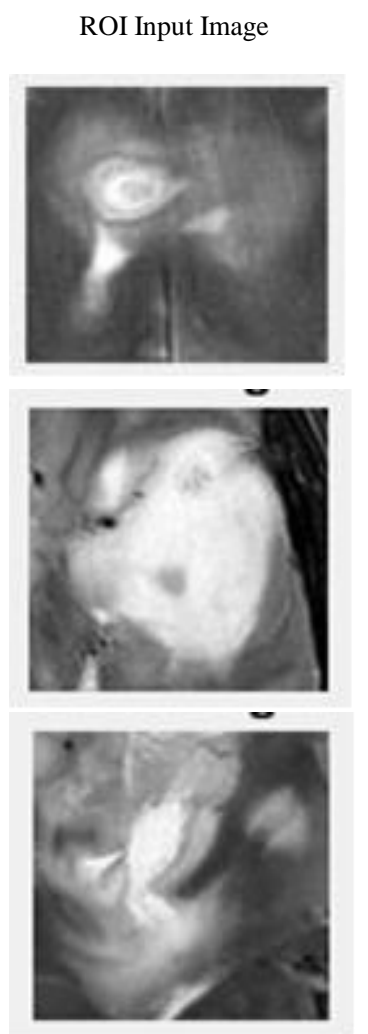

$8(b)$
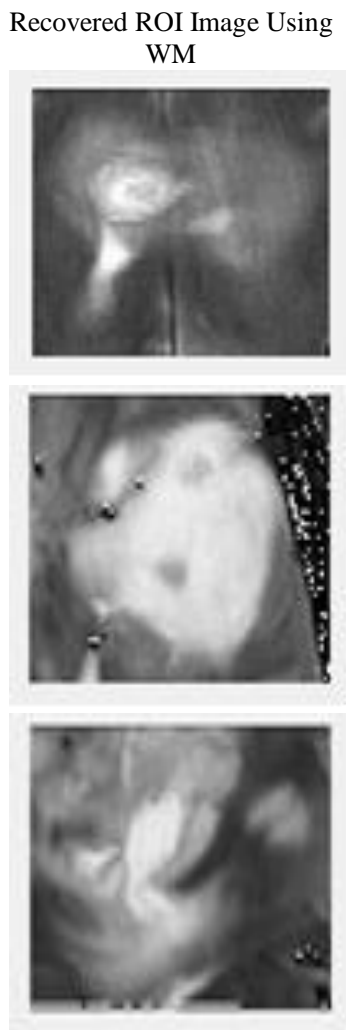

$8(\mathrm{c})$
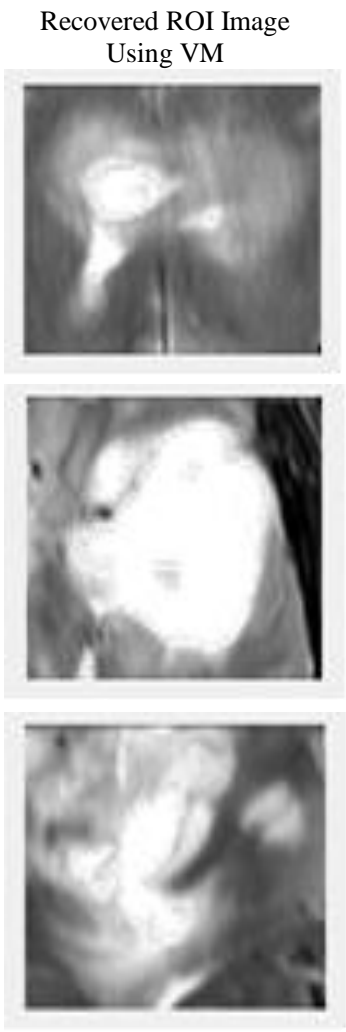

$8(d)$

Figure 8. ROI- Based DWT using Wallace-tree Multiplier (WM) and Vedic Multiplier (VM) Results on FPGA Platform: 8(a) Different Brain-MRI input images, 8(b) ROI input image, 8(c) Recovered ROI Image using WM, 8(d) Recovered ROI Image using VM

Table 2 shows the PSNR and MSR of both ROI Based DWT-WM and VM after recovery. The image-2 of DWT-VM with ROI size 90x90 gives better PSNR with $31.491 \mathrm{~dB}$ and MSE ratio of 46.12 concerning DWT-WM method includes the PSNR of 27.91 and MSE of 105.2.

Table 2. Observation of PSNR and MSE for ROI based DWT-WMand DWT-VM after compression and reconstruction

\begin{tabular}{cccccc}
\hline $\begin{array}{c}\text { Brain MRI Input- } \\
\text { Images }\end{array}$ & \multirow{2}{*}{ ROI-Image Size } & \multicolumn{2}{c}{ ROI-DWT-WM } & \multicolumn{2}{c}{ ROI-DWT-VM } \\
& & PSNR $(\mathrm{dB})$ & MSE & PSNR (dB) & MSE \\
\hline 1 & $86 \times 90$ & 27.156 & 125.64 & 28.506 & 91.7 \\
2 & $90 \times 90$ & 27.91 & 105.2 & 31.491 & 46.12 \\
3 & $90 \times 86$ & 27.23 & 122.9 & 30.024 & 64.65 \\
\hline
\end{tabular}

Figure 9 shows the comparative graph of both ROI-Based DWT-WM and VM methods of three brain images for PSNR and MSE respectively. The ROI-Based DWT-VM gives on an average PSNR of $30.007 \mathrm{~dB}$ than DWT-WM with average PSNR of 27.43 concerning three brain images with an improvement of $8.58 \%$. Similarly, The ROI-Based DWT-VM gives on an average MSE of 67.49 than DWT-WM with average MSE of 117.91 concerning three brain images with an improvement of $42.76 \%$ and which is quite acceptable as lossless image quality. 

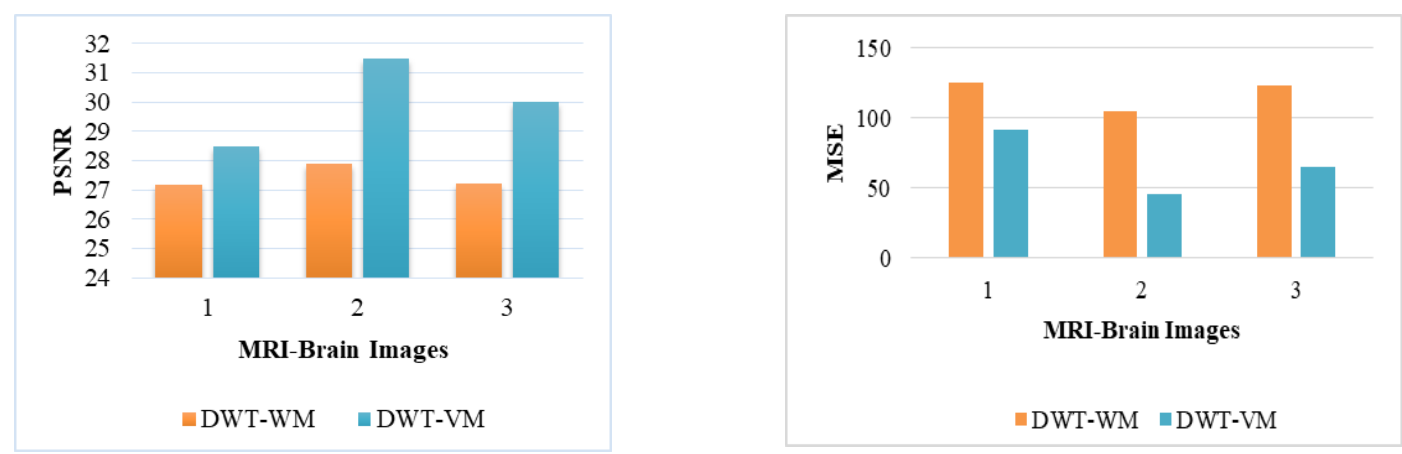

Figure 9. Comparative results of PSNR and MSR for Three different Brain-MRI images using ROI-based DWT-WM and DWT-VM.

The area resource of the Wallace tree multiplier uses 87 slice LUT's which less than the Vedic Multiplier of 109 slice LUT's. The Vedic multiplier consumes the 7.519nsec for path delay and is less than the Wallace tree multiplier of 9.009nsec as tabulated in Table 3.

Table 3. Resource and delay obtained of Wallace-tree Multiplier (WM)

\begin{tabular}{lcc}
\multicolumn{3}{c}{ and Vedic Multiplier (VM) on FPGA } \\
\hline Area and Delay utilization & Vedic Multiplier & Wallace-Tree Multiplier \\
\hline Number of Slice LUTs & 109 & 87 \\
Path Delay (ns) & 7.519 & 9.009 \\
\hline
\end{tabular}

The resource utilization (Area), timing and power consumption of ROI-based DWT-WM and DWTVM on FPGA platform is tabulated in Table 4 and Table 5 respectively. It shows that the DWT-WM consumes fewer area resources includes slice registers, LUT's, LUT-FF pairs than DWT-VM. Similarly, for timing and power utilization, ROI-based DWT-WM is better than DWT-VM. The ROI-based DWT-WM method works high speed of $175.199 \mathrm{MHz}$ and utilizes less power of 0.099Wover DWT-VM Method.

Table 4. Resource utilization of ROI-based DWT-WM and DWT-VM on FPGA

\begin{tabular}{llll}
\hline Logic Utilization & Available & DWT-WM & DWT-VM \\
\hline Number of Slice Registers & 126800 & 115 & 141 \\
Number of Slice LUTs & 63400 & 243 & 276 \\
LUT-Flip-flop (FF) pairs & 363 & 66 & 54 \\
\hline
\end{tabular}

Table 5. Timing and power consumption of ROI-based DWT-WM and DWT-VM on FPGA

\begin{tabular}{|c|c|c|c|c|c|}
\hline Timing Utilization & DWT-WM & DWT-VM & Power Utilization & DWT-WM & DWT-VM \\
\hline Minimum period (ns) & 5.708 & 6.016 & Total Power(W) & 0.099 & 0.103 \\
\hline Maximum Frequency $(\mathrm{MHz})$ & 175.199 & 166.21 & Dynamic Power (W) & 0.017 & 0.023 \\
\hline
\end{tabular}

\section{CONCLUSION}

This research paper has presented a cost-effective technique for image compression of MRI-Brain images. The proposed design includes ROI- Based DWT using Wallace-tree multiplier and Vedic multiplier. The 9/7 Lifting scheme used for the DWT method, 8X8 Vedic multiplier is constructed using 4x4 Vedic multiplier and adders. The Wallace-tree (WM) Multiplier is designed using booth encoding and compressors along with adder-tree modules. The 1D-DWT results and ROI image Reconstruction hardware Results using DWT-WM and DWT-VM presented in the results section. For image quality and resolution, DWT-VM method gives better PSNR and MSE with an improvement of $8.58 \%$ and $42.76 \%$ respectively over DWTWM method with promising lossless image compression characteristics. Concerning Chip hardware constraints, the DWT-WM method utilizes less resource Area, high operating frequency, and less power consumption than DWT-VM Method with optimal fast computation on FPGA. 


\section{REFERENCES}

[1] Scholl, Ingrid, TilAach, Thomas M. Deserno, and TorstenKuhlen. "Challenges of medical image processing," Computer science-Research and development 26, no. 1-2 (2011): 5-13, Springer.

[2] Kavitha, S., and R. J. Anandhi. "A survey of image compression methods for low depth-of-field images and image sequences," Multimedia Tools and Applications 74, no. 18 (2015): 7943-7956. Springer.

[3] Singh, Abhipriya, and K. G. Kirar. "Review of image compression techniques," Recent Innovations in Signal processing and Embedded Systems (RISE), 2017 International Conference on. IEEE, 2017.

[4] Bhade, Uday, et al. "Comparative study of DWT, DCT, BTC and SVD techniques for image compression," Recent Innovations in Signal processing and Embedded Systems (RISE), 2017 International Conference on. IEEE, 2017.

[5] Mantoro, Teddy, and FifitAlfiah. "Comparison methods of DCT, DWT and FFT techniques approach on lossy image compression," Computing, Engineering, and Design (ICCED), 2017 International Conference on. IEEE, 2017.

[6] Ashwini, K., R. Amutha, and K. Harini. "Sparse based image compression in wavelet domain," Signal Processing and Communication (ICSPC), 2017 International Conference on. IEEE, 2017.

[7] Raghavendra, C., S. Sivasubramanian, and A. Kumaravel. "Improved image compression using effective lossless compression technique," Cluster Computing: 1-6, Springer.

[8] Suganya, S. D., M. Rajasekhar Reddy, and N. MadhuSudana Rao. "Increasing the quality of reconstructed image through hybrid compression technique," 2017 International Conference on Energy, Communication, Data Analytics and Soft Computing (ICECDS). IEEE, 2017.

[9] Reddy, B. Venkateswara, P. Bhaskara Reddy, P. Sateesh Kumar, and A. Sateesh Reddy. "Lossless compression of medical images for better diagnosis," In Advanced Computing (IACC), 2016 IEEE 6th International Conference on, pp. 404-408. IEEE, 2016.

[10] Sengupta, Anirban, and Dipanjan Roy. "Mathematical Validation of HWT Based Lossless Image Compression," Nanoelectronic and Information Systems (iNIS), 2017 IEEE International Symposium on. IEEE, 2017.

[11] Devadoss, C. Peter, and B. Sankaragomathi. "Near lossless medical image compression using block BWT-MTF and hybrid fractal compression techniques," Cluster Computing (2018): 1-9, Springer.

[12] Grailu, Hadi. "Textual image compression at low bit rates based on region-of-interest coding," International Journal on Document Analysis and Recognition (IJDAR) 19, no. 1 (2016): 65-81.

[13] Sridhar, V. "VCAR: Vedic Compression Algorithm over Region of interest on radiological image," In Emerging Research in Electronics, Computer Science and Technology (ICERECT), 2015 International Conference on, pp. 137-142. IEEE, 2015.

[14] Kumar, J. Vinoth, and C. Kumar Charlie Paul. "Design of modified vedic multiplier and FPGA implementation in multilevel 2d-DWT for image processing applications," In Current Trends in Engineering and Technology (ICCTET), 2014 2nd International Conference on, pp. 508-511. IEEE, 2014.

[15] Hamzah, Fairoza Amira Binti, Taichi Yoshida, and Masahiro Iwahashi. "Four-dimensional image compression with region of interest based on non-separable double lifting integer wavelet transform," Asia-Pacific Signal and Information Processing Association Annual Summit and Conference (APSIPA ASC), 2017. IEEE, 2017.

[16] Chakraborty, Srija, Ayan Banerjee, Sandeep KS Gupta, and Philip R. Christensen. "Region of interest aware compressive sensing of THEMIS images and its reconstruction quality," In 2018 IEEE Aerospace Conference. IEEE, 2018

[17] Shakya, Amit Kumar, AyushmanRamola, and Deepak Chander Pandey. "Polygonal region of interest-based compression of DICOM images," Computing, Communication and Automation (ICCCA), 2017 International Conference on. IEEE, 2017.

[18] Yee, David, Sara Soltaninejad, DeborsiHazarika, Gaylord Mbuyi, Rishi Barnwal, and AnupBasu. "Medical image compression based on region of interest using better portable graphics (BPG)," In Systems, Man, and Cybernetics (SMC), 2017 IEEE International Conference on, pp. 216-221. IEEE, 2017.

[19] Kanakatte, Aparna, Rakshith Subramanya, Ashik Delampady, RajaramaNayak, Balamuralidhar Purushothaman, and JayavardhanaGubbi. "Cloud solution for histopathological image analysis using region of interest-based compression," In Engineering in Medicine and Biology Society (EMBC), 2017 39th Annual International Conference of the IEEE, pp. 1202-1205. IEEE, 2017.

[20] Maye, G. Kiran, and T. Srinivasulu. "VLSI architecture for coherent 9/7 lifting based 2D-discrete wavelet transform," In India Conference (INDICON), 2015 Annual IEEE, pp. 1-6. IEEE, 2015.

[21] Bhosale, Nayna Vijaykumar, and Sudhir S. Kanade. "2D DWT lifting image compression scheme for error tolerant applications," 2017 International Conference on Intelligent Sustainable Systems (ICISS). IEEE, 2017.

[22] Swami, Shweta S., and Altaf O. Mulani. "An efficient FPGA implementation of discrete wavelet transform for image compression," 2017 International Conference on Energy, Communication, Data Analytics and Soft Computing (ICECDS). IEEE, 2017.

[23] Gadakh, S. N., and A. S. Khade. "FPGA implementation of high speed vedic multiplier," (2016): 4-4.

[24] Bandi, VijayaLakshmi. "Performance analysis for Vedic multiplier using modified full adders," In Power and Advanced Computing Technologies (i-PACT), 2017 Innovations in, pp. 1-5. IEEE, 2017.

[25] Kahar, DravikKishorBhai, and Harsh Mehta. "High speed vedic multiplier used vedic mathematics," In Intelligent Computing and Control Systems (ICICCS), 2017 International Conference on, pp. 356-359. IEEE, 2017.

[26] Luu, Xuan-Vy, Trong-Thuc Hoang, Trong-Tu Bui, and Anh-Vu Dinh-Duc. "A high-speed unsigned 32-bit multiplier based on booth-encoder and wallace-tree modifications," In Advanced Technologies for Communications (ATC), 2014 International Conference on, pp. 739-744. IEEE, 2014.

An efficient design approach of ROI based DWT using vedic and wallace tree multiplier on ... (Vijaya S.M.) 
[27] Asif, Shahzad, and Yinan Kong. "Design of an algorithmic Wallace multiplier using high speed counters," In Computer Engineering \& Systems (ICCES), 2015 Tenth International Conference on, pp. 133-138. IEEE, 2015.

[28] De Castro, Lucas Gaia, Henrique Seiti Ogawa, and Bruno de CarvalhoAlbertini. "Automated Generation of HDL Implementations of Dadda and Wallace Tree Multipliers," In Computing Systems Engineering (SBESC), 2017 VII Brazilian Symposium on, pp. 17-22. IEEE, 2017.

[29] Ram, G. Challa, D. Sudha Rani, R. Balasaikesava, and K. BalaSindhuri. "Design of delay efficient modified 16 bit Wallace multiplier," In Recent Trends in Electronics, Information \& Communication Technology (RTEICT), IEEE International Conference on, pp. 1887-1891. IEEE, 2016.

[30] Kamal, Sunaina, and Channpreet Kaur. "Design of Vedic Multiplier in Image Compression and FPGA Implementation Using Discrete Wavelet Transform (DWT) Algorithm," International Journal of Engineering Science 2259, 2016.

[31] Suma, and V. Sridhar. "ROI-based Compression on Radiological Image by Urdhva-Tiryagbhyam and DWT Over FPGA," International Journal of Advanced Computer Science and Applications, Vol. 8, No. 2, 211-218, 2017.

[32] Jufriadif Na'am, Johan Harlan, Irawadi Putra, Romi Hardianto, Mutiana Pratiwi, "An Automatic ROI of the Fundus Photography," International Journal of Electrical and Computer Engineering (IJECE), Vol. 8, No. 6, 2017

[33] Lakshminarayana, M., and Mrinal Sarvagya. "MICCS: A Novel Framework for Medical Image Compression using Compressive Sensing." International Journal of Electrical and Computer Engineering (IJECE), Vol. 8, No. 5, 2018 .

\section{BIOGRAPHIES OF AUTHORS}
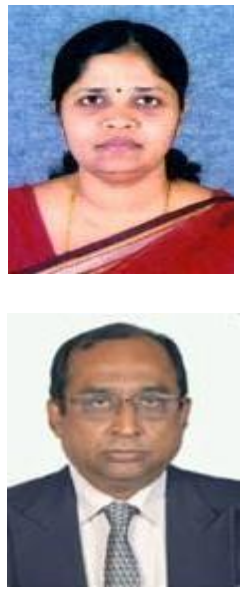

Vijaya S.M obtained B. E in Electronics \& Communication Engineering from Gulbarga University \& M. Tech in Applied Electronics from Sathyabhama University Chennai. Presently she is working as Associate Professor in Electronics \& Communication Engineering Department of RajaRajeswari College of Engineering, Bangalore. She has altogether 17 years of Teaching experience. She is presently pursuing $\mathrm{PhD}$ at VTU, Belgavi. She has published 6 International journal papers \& 8 Conference papers. She has guided PG \& UG Projects. She is a Life member of ISTE \& IETE. Her areas of interest are Signals \& Systems, Image Processing, VLSI.

Dr.K. Suresh obtained his B.E., M.E., \& M. Tech from university of Mysore \& PhD from Kuvempu university. Presently working as Principal SEA College of Engineering \& Technology. He has over 30 years of Teaching experience. Currently he is guiding 6 students in VTU. His area of interest are Signals \& Systems, Digital Signal Processing, Image Processing, Multirate signal processing, VLSI for DSP, Spectrum Analysis. He has published over 15 International journal papers. 